\title{
Desarrollo de un Indicador de la Calidad del Agua usando Estadística Aplicada, Caso de Estudio: Sulocuenca Zanjón Oscuro
}

\author{
Jefferson Valdes-Basto ${ }^{1}$ \\ Natalia E. Samboni-Ruiz ${ }^{2}$ \\ Yesid Carvajal-Escobar ${ }^{3}$
}

\section{Resumen}

Las aguas de la subcuenca Zanjón Oscuro (Departamento del Cauca), son utilizadas para regar caña de azúcar, mediante el uso de drenajes artificiales. En su recorrido el cauce recibe aguas residuales domésticas e industriales de la población y la industria azucarera, lo cual ha originado la contaminación del mismo. Se presenta la evaluación de la calidad del agua mediante la elaboración de un índice de calidad del agua "ICA", a partir del uso de la Estadística Aplicada. Se propone el uso de una metodología basada en curvas de calidad de diferentes parámetros fisicoquímicos desarrollados a partir de encuestas realizadas a varios expertos, con el objeto de establecer límites cualitativos y cuantitativos de la calidad del agua; para posteriormente seleccionar la curva óptima que refleje la mejor decisión, en dicha evaluación. Los resultados fueron comparados con los indicadores de contaminación del agua (ICO$\mathrm{MO}$ ). Finalmente se obtuvieron dos indicadores: el primero conformado por las variables oxígeno disuelto, demanda bioquímica de oxígeno y coliformes fecales, que son indicadores de la contaminación por materia orgánica. El segundo indicador incluye la demanda bioquímica de oxígeno, $\mathrm{pH}$, conductividad, fosfatos, nitratos,

1 Grupo IREHISA, Escuela de Ingeniería de Recursos Naturales y del Ambiente EIDENAR, Universidad del Valle, jeffer.valdes@gmail.com

2 Grupo IREHISA, Escuela de Ingeniería de Recursos Naturales y del Ambiente EIDENAR, Universidad del Valle, nasquim@yahoo.es

3 Grupo IREHISA, Escuela de Ingeniería de Recursos Naturales y del Ambiente EIDENAR, Universidad del Valle, yecarvaj@univalle.edu.co

Fecha de recepción: 17 de Noviembre de 2010

Fecha de aceptación: 30 de Julio de 2011 
turbiedad y sólidos totales, que permiten una evaluación general de la calidad del agua.

\section{Palabras clave}

Índice de calidad del agua (ICA), contaminación del agua, estadística aplicada, curvas de valoración.

\section{Abstract}

The waters of the subriver basin Zanjón Oscuro, located in the plains of northern Cauca, Cauca Department, are used for irrigation of sugar cane by the use of artificial drainages. In its course the stream receives domestic and industrial sewage from the population and the sugar industry, which has led to pollution of it. This paper presents the work the evaluation of the quality of the water by means of the elaboration of water quality index "WQI", from the use of the Applied Statistic. For this aim the use of a methodology based on the elaboration of curves of quality of different physicochemical parameters sets out that they were developed from a realised survey to different experts, with the intention of establishing qualitative and quantitative limits of the quality of the water; later to select the optimal curve that reflects the best decision to evaluate the quality of the water. The results were compared with the indicators of contamination of the water. Obtaining like result two indicators, the first conformed by the variables dissolved oxygen, biochemical demand of oxygen and faecal coliforms, which are indicating of the contamination by organic matter. The second includes the biochemical demand of oxygen, $\mathrm{pH}$, conductivity, phosphates, nitrates, turbidity and total solids, which allow a general evaluation of the quality of the water.

\section{Keywords}

Index of quality of water (IWQ), contamination of the water, applied statistic, rating curves. 


\section{INTRODUCCIÓN}

En la valoración y evaluación de la calidad del agua se han empleado diversas metodologías entre las que se incluyen: comparación de las variables con la normatividad vigente; los indicadores ICA donde a partir de un grupo de variables medidas se genera una valor que califica y cualifica la fuente y metodologías más elaboradas como la modelación (Samboni, 2009).

Desde la aparición de los indicadores ICA en la década del 60 con Horton (1965) y Liebman (1969), se ha buscado unificar la metodología para su determinación; en la actualidad se basa en el desarrollo de tres pasos fundamentales: selección de parámetros, determinación del subíndice para cada parámetro y fórmula de agregación de los subíndices, donde la estadística ha jugado un papel importante en la construcción y análisis de este tipo de información (Abbasi, 2002; Samboni et. al. 2007).

Este trabajo plantea la construcción de un índice ICA usando la Estadística Aplicada mediante la elaboración de curvas de calidad, que se obtienen a partir de encuestas realizadas a cuatro grupos que trabajan en la evaluación de la calidad del agua, se fijaron límites cualitativos y cuantitativos de diferentes variables fisicoquímicas que fueron incluidas en los indicadores propuestos, ICA-Orgánico e ICA-General. El primero utiliza las variables de oxígeno disuelto $(\mathrm{OD})$, demanda bioquímica de oxígeno $\left(\mathrm{DBO}_{5}\right)$ y coliformes fecales; mientras que el segundo, usa $\mathrm{pH}$, conductividad, demanda bioquímica de oxígeno (DQO), fosfatos, nitratos, turbiedad y sólidos totales (ST).

Los resultados obtenidos fueron comparados con dos indicadores consultados en literatura y que son ampliamente utilizados en Colombia y en el mundo, como son: ICA-NSF propuesto por Fundación Nacional de Saneamiento de los Estados Unidos (NSF, 2006) y el índice de contaminación del agua ICOMO (Ramírez \& Viña 1998); éste último, elaborado en el país y desarrollado a través de técnicas multivaridas (Análisis de Componentes Principales, ACP), (Hair et al, 1999; Peña, 2002). 


\section{METODOLOGíA}

\subsection{Descripción de la Zona de Estudio}

La zona de estudio corresponde a una llanura plana aluvial que no presenta zonas de erosión en el recorrido del cauce, entre los $1095 \mathrm{msnm}$ y $1100 \mathrm{msnm}$; está localizada en la zona norte del Departamento del Cauca y pertenece a la cuenca alta del río Cauca. Las aguas de la subcuenca son distribuidas principalmente en zanjones y acequias para regar los cultivos de caña de azúcar que predominan en la región, formando una serie de drenajes artificiales. La acequia Santa Ana, el Zanjón Rico y el propio Zanjón Oscuro hacen parte del sistema hídrico artificial (CRC-FONAN, 2000).

Para la evaluación de la calidad del recurso mediante el uso de los indicadores ICA e ICO se utilizaron los reportes de las variables fisicoquímicas y bacteriológicas del agua a lo largo de la subcuenca durante los años 2005 y 2006, obtenidas por la Corporación Autónoma Regional del Cauca (CRC), donde se incluyen 5 estaciones sobre el cauce principal de la subcuenca Zanjón Oscuro (Fig.1).

Los puntos de monitoreo de agua son: Estación 1, La acequia Santa Ana antes del vertimiento del agua residual domestica (ARD). Estación 2, La Acequia Santa Ana después de la descarga de aguas residuales industriales. Estación 3, Zanjón Oscuro después del Ortigal. Estación 4, Antes de la descarga del zanjón La Secreta y la Estación 5, desembocadura al río Cauca. Para obtener el ICA General y el ICA Orgánico para la subcuenca de Zanjón Oscuro se siguieron las siguientes etapas: desarrollo de las curvas de valoración, fórmula de agregación y determinación del ICAGeneral y el ICA-Orgánico. A continuación se realiza una breve descripción de cada una.

\subsection{Curvas de Valoración}

Para la elaboración de las curvas de valoración se diseñó una encuesta que fue aplicada realizada por dos grupos de investigación de la Universidad del Valle, la Universidad de Antioquia y la Corporación Autónoma Regional del Cauca (CRC), cuyo objetivo consistió en generar un determinado número de rangos para cada 
una de las variables, cada uno de ellos asociado en general a cuatro condiciones de calidad: Excelente, Buena, Regular y Mala.

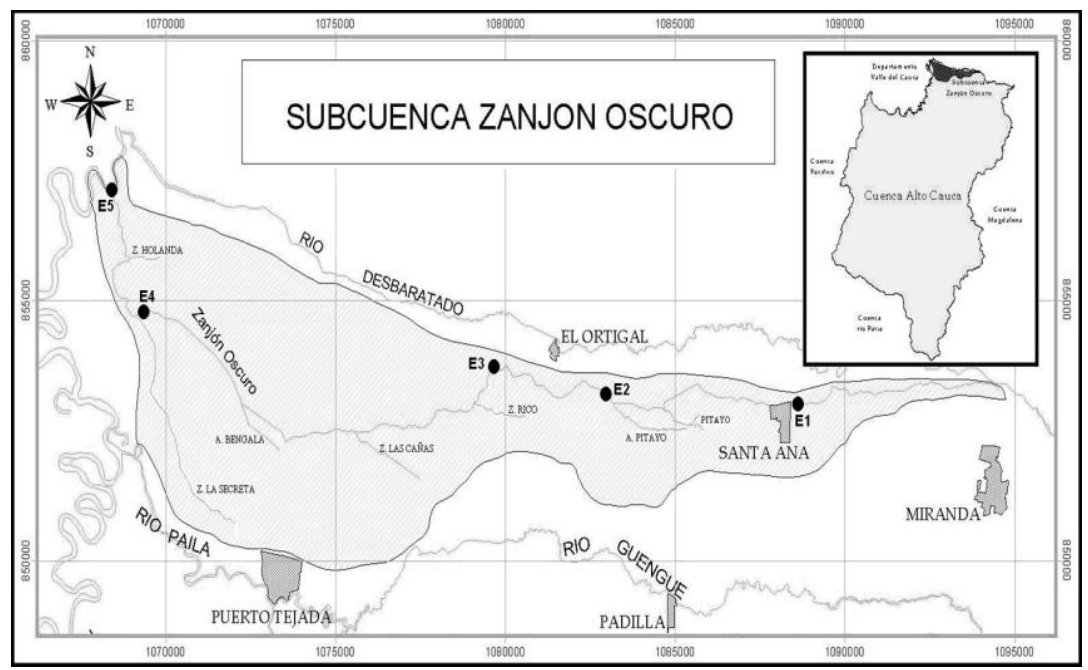

Fig. 1. Mapa de la subcuenca da Zanjón Oscuro

A cada una de las valoraciones se les asignó una valor cuantitativo y cualitativo definido entre cero y el valor unitario; esto es, si la función genera un valor menor que 0,50 entonces las condiciones de calidad serían calificadas como mala, mientras que si está entre 0,50 y menor a 0,75 será regular; entre 0,75 y 0,90 buena y si es mayor que 0,90 el calificativo asignado será excelente. El número de curvas de valoración obtenidas coincidió con el número de encuestas diligenciadas, más la cantidad de funciones que han sido definidas en estudios previos; esto significa que asociado al valor particular de una variable, se obtuvieron un conjunto de valores derivados de cada una de las curvas de valoración obtenidas, valores que fueron tenidos en cuenta en su totalidad.

Además, en este estudio, se partió del principio básico de no homogeneidad en las opiniones de los expertos evaluados, es decir, se consideró la mediana de estos valores como una opinión intermedia y el rango (máximo y mínimo) como representante de la variabilidad o medida de discrepancia entre estas opiniones. Los valores de mediana, mínimo y máximo fueron tenidos en cuenta 
para todas las variables y de esta forma se definió un rango de valores para el ICA, considerando nuevamente la heterogeneidad de los expertos. De esta evaluación se seleccionó la que mejor representó el criterio de evaluación de la calidad del agua.

\subsection{Selección de Variables}

La elección de variables depende en gran medida del criterio del experto como también de la información existente, los criterios de tiempo, localización y su importancia como estándar de calidad (Ball \& Church, 1980; IDEAM, 2004). Para el caso de Zanjón Oscuro se realizó un análisis general de las variables calificándolas en dos grupos, de la siguiente manera: $\mathrm{OD}, \mathrm{DBO}_{5}$ y coliformes fecales, asociadas a la contaminación por materia orgánica; $\mathrm{pH}$, conductividad, DQO, fosfatos, nitratos, turbiedad y ST, permiten una evaluación general de la calidad del agua.

\subsection{Formula de Agregación y Pesos}

La fórmula de agregación para el cálculo del índice ICA se determinó mediante dos métodos: la media aritmética ponderada y la media geométrica ponderada, que son las fórmulas más utilizadas en la construcción de los ICAs (Van Helmond \& Breukel, 1997; Abbasi, 2002). La Tabla 1 relaciona cada una de estas fórmulas, las variables empleadas y el peso asignado a cada una de ellas, el cual se aplica a la mejor tendencia (mediana, máximo o mínimo) que se seleccionó anteriormente.

Con el fin de valorar los indicadores propuestos, se compararon los resultados con dos fórmulas propuestas (promedio aritmético ponderado (1) y (2); y promedio geométrico ponderado (3) y (4)) comparadas con las formulas existentes y que son ampliamente utilizados por agencias de control de la calidad del agua, como son: el ICA-NSF, elaborado por la Fundación Nacional de Saneamiento de los Estados Unidos (NSF 2006) y el ICOMO, indicador desarrollado en Colombia por Ramírez \& Viña (1998). 
Tabla 1. Fórmulas de agregación en la construcción del ICA

\section{ICA Formula de Agregación}

$I C A=\sum_{i=1}^{n} w_{i} *\left(I\left(X_{i}\right)\right)$

$w_{i}$ : Peso de cada variable

$\mathrm{pH}=0,2 ;$ conductividad $=0,1 ; \mathrm{DQO}=0,15$, fosfatos $=0,15$;

nitratos $=0,2$; turbiedad $=0,1 ;$ sólidos totales $=0,1$

$I\left(X_{i}\right)$ : valoración obtenida a partir de la curva para cada variable $X_{i}$

$I C A=\sum_{i=1}^{n} w_{i} *\left(I\left(X_{i}\right)\right)$

$w_{i}$ : Peso de cada variable

Oxígeno disuelto $=0,3 ; \mathrm{DBO}=0,4 ;$ Coliformes fecales $=0,3$

$I\left(X_{i}\right)$ : valoración obtenida a partir de la curva para cada variable $X_{i}$

$$
I C A=\prod_{i=1}^{n}\left(\left(I\left(X_{i}\right)\right)^{w_{i}}\right.
$$

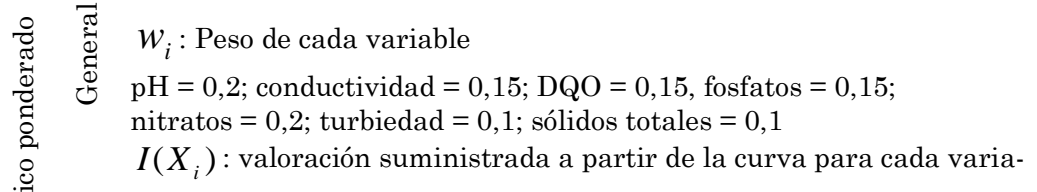

ble $X_{i}$

$$
I C A=\prod_{i=1}^{n}\left(\left(I\left(X_{i}\right)\right)^{w_{i}}\right.
$$

$w_{i}$ : Peso de cada variable

Oxígeno disuelto $=0,3 ; \mathrm{DBO}=0,4 ;$ Coliformes fecales $=0,3$

$I\left(X_{i}\right)$ : valoración obtenida a partir de la curva para cada variable $X_{i}$ 


\section{RESULTADOS Y DISCUSION}

\subsection{Curvas de Valoración}

En la Fig. 2 se observa el comportamiento de la variable de $\mathrm{pH}$, de la encuesta realizada a las entidades, donde se pueden calificar la calidad del agua bajo tres juicios: pesimista que corresponde al valor mínimo de los encuestados, optimista que corresponde al máximo valor para los encuestados, e intermedia, que resultaría de la mediana entre estos dos valores, ésta última representa la tendencia central, la cual permite discriminar las opiniones extremas que pudiese haber tenido algún experto encuestado.

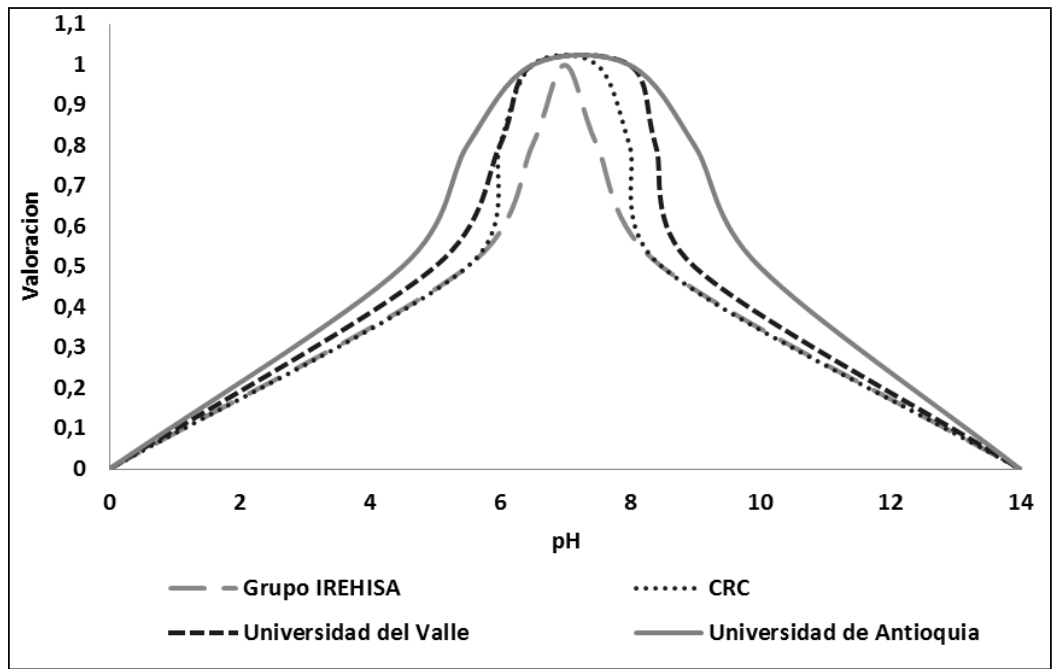

Fig. 2. Curvas de valoración para el $\mathrm{pH}$

Este procedimiento se realizó exactamente igual para cada una de las variables y se estableció que la mediana, de todas las mediciones, sea la curva que mejor representa la estadística de las encuestas, consolidando la información y permitiendo evaluar cada una de las variables de la calidad del agua, como son: $\mathrm{pH}, \mathrm{DBO}_{5}$, coliformes fecales, conductividad, DQO, fosfatos, nitratos, turbiedad y sólidos totales. 


\subsection{Selección de Variables}

Debido al gran contenido de aguas residuales domésticas que llegan a la corriente principal provenientes de la zona urbana de Puerto Tejada y corregimiento Santa Ana, se evaluaron las variables de $\mathrm{OD}, \mathrm{DBO}_{5}$ y coliformes fecales, como indicadoras de contaminación por materia orgánica, encontrando que en la estación uno se encuentra mayor cantidad de oxígeno disuelto y en las demás estaciones ocurre una disminución del mismo, observando que las condiciones cambian respecto a los otros puntos de monitoreo, evidenciando el impacto en la calidad del recurso debido a las aguas residuales provenientes de los centros poblados (Fig. 3).

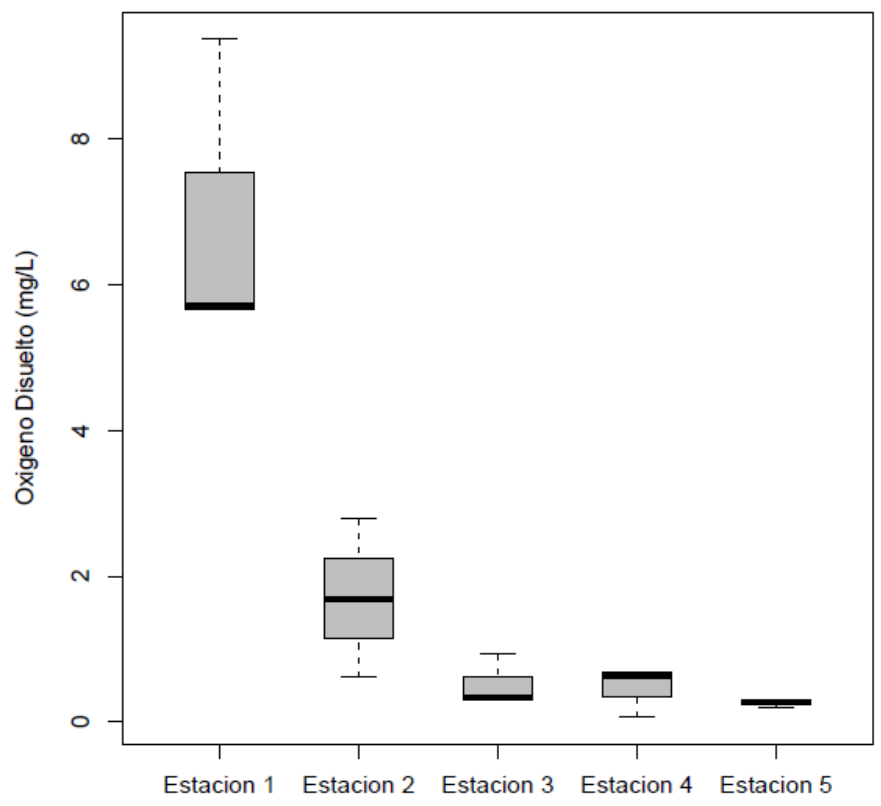

Fig. 3. Diagrama de cajas y alambres del oxígeno disuelto

Este comportamiento es similar para el contenido de $\mathrm{DBO}_{5}$, mientras que en el caso de las coliformes fecales se incrementan a limites superiores a las $2000 \mathrm{NMP} / 100 \mathrm{~mL}$, valores que están por encima de los sugeridos por el Decreto 3930/2010. Encontrando 
que los tres parámetros pueden ser usados como indicadores de contaminación del agua por materia orgánica en el indicador "ICAOrgánico".

La evaluación del recurso en forma general se realizó mediante el "ICA-General", que incluye las variables de $\mathrm{pH}$, conductividad, DQO, Fosfatos, Nitratos, Turbiedad y ST. Encontrando que el pH en todas las estaciones se mantiene en valores que oscilan entre 8,2-5,5; los cuales son permitidos para diversos usos en el Decreto 3930/2010. Entre la turbiedad y los ST, hay una estrecha relación, donde a partir de la estación 2 se muestra un incremento significativo en comparación con el resto de estaciones; esto debido a la gran cantidad de iones y sólidos en suspensión que son aportados por las aguas residuales domesticas e industriales, el comportamiento de las variables en las estaciones se observa en la Fig. 4.
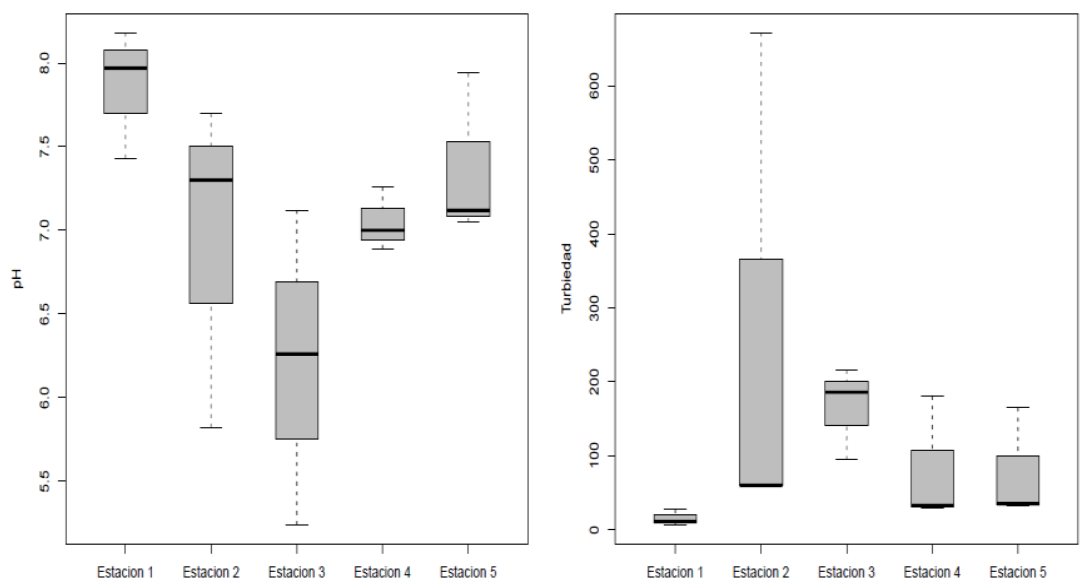

Fig. 4. Diagrama de cajas y alambres del pH (izquierda) y la turbiedad (derecha)

La evaluación del contenido de materia no biodegradable, mediante la relación $\mathrm{DBO}_{5} / \mathrm{DQO}$ (Fig. 5), indica que en las tres primeras estaciones hay un contenido de material inorgánico no biodegradable, que puede provenir de residuos de aguas residuales industriales o domésticas. 


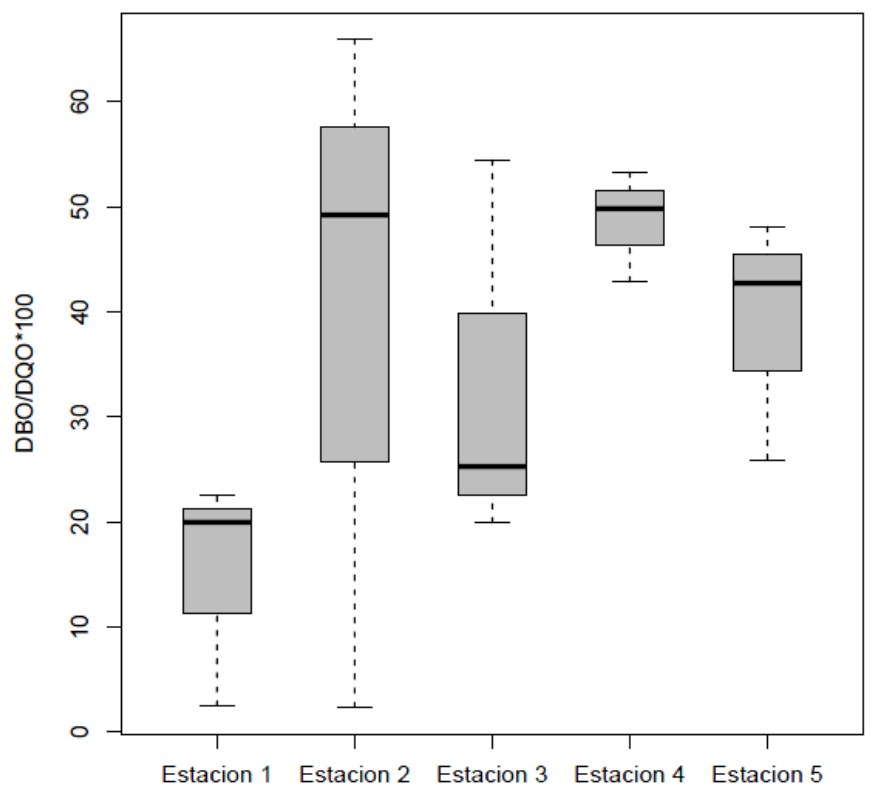

Fig. 5. Comportamiento $\mathrm{DBO}_{5} / \mathrm{DQO}$

La Tabla 2 muestra algunos estadísticos descriptivos (media, desviación estándar, coeficiente de variación) de las variables de estudio en todas las estaciones. El coeficiente de variación es un indicador que mide el grado de homogeneidad de los datos, en este caso solo el $\mathrm{pH}$ muestra un valor de coeficiente de variación bajo, lo cual indica su poca variabilidad entre estaciones de monitoreo, en el resto de las variables se encontraron valores (coeficientes de variación) muy altos, y en consecuencia mucha variabilidad entre estaciones de monitoreo para cada una de las variables (Hernández \& Ferri, 2004; Martínez, 2006; Romero \& Zúnica, 2008).

\subsection{Formula de Agregación}

Al aplicar las dos fórmulas de agregación se observó que habían valoraciones de cero, por lo que al usar el promedio geométrico ponderado el resultado tiene a cero, por lo tanto se usó el promedio aritmético ponderado. La Tabla 3 presenta los resultados al 
aplicar los ICAs propuestos y compararlos con los indicadores de literatura ICA-NSF e ICOMO.

Tabla 2. Estadísticas descriptivas de las variables de estudio Zanjón Oscuro

\begin{tabular}{lccc}
\hline & Media & $\begin{array}{c}\text { Desviación } \\
\text { estándar }\end{array}$ & $\begin{array}{c}\text { Coeficiente de } \\
\text { variación }\end{array}$ \\
\hline OD $(\mathrm{mg} / \mathrm{L})$ & 1,97 & 2,77 & 1,40 \\
$\mathrm{pH}$ & 7,1 & 0,8 & 0,11 \\
$\mathrm{DBO}_{5}(\mathrm{mg} / \mathrm{L})$ & 135 & 163 & 1,20 \\
$\mathrm{DQO}_{(\mathrm{mg} / \mathrm{L})}$ & 345 & 428 & 1,24 \\
Turbiedad $(\mathrm{UNF})$ & 121 & 168 & 1,39 \\
Conductividad $(\mu \mathrm{S} / \mathrm{cm})$ & 419 & 180 & 0,43 \\
Fosfatos $(\mathrm{mg} / \mathrm{L})$ & 10,5 & 12,6 & 1,20 \\
Nitratos $(\mathrm{mg} / \mathrm{L})$ & 5,0 & 5,7 & 1,13 \\
ST $(\mathrm{mg} / \mathrm{L})$ & 333 & 178 & 0,53 \\
Coliformes fecales $(\mathrm{NMP} / 100 \mathrm{~mL})$ & 16958 & 50686 & 2,99 \\
\hline
\end{tabular}

Tabla 3. Resultados de los de ICAs e ICOs para Zanjón Oscuro

\begin{tabular}{|c|c|c|c|c|c|c|c|}
\hline \multirow{2}{*}{ Estación } & \multirow{2}{*}{ Fecha } & \multirow{2}{*}{ ICA-NSF } & \multirow{2}{*}{ ICOMO } & \multicolumn{2}{|c|}{ ICA-General } & \multicolumn{2}{|c|}{ ICA-Orgánico } \\
\hline & & & & Aritm. & Geom. & Aritm. & Geom. \\
\hline \multirow{4}{*}{1} & Sep-2005 & Buena & Buena & Buena & Buena & Regular & Mala \\
\hline & Mar-2006 & Buena & Buena & Buena & Regular & Regular & Regular \\
\hline & Jul-2006 & Buena & Buena & Buena & Buena & Regular & Regular \\
\hline & Sep-2005 & Mala & Mala & Mala & Mala & Mala & Mala \\
\hline \multirow[t]{2}{*}{2} & Mar-2006 & Mala & Mala & Mala & Mala & Mala & Mala \\
\hline & Jul-2006 & Mala & Regular & Mala & Mala & Mala & Mala \\
\hline \multirow{3}{*}{3} & Sep-2005 & Mala & Mala & Mala & Mala & Mala & Mala \\
\hline & Mar-2006 & Mala & Mala & Mala & Mala & Mala & Mala \\
\hline & Jul-2006 & Mala & Regular & Mala & Mala & Mala & Mala \\
\hline \multirow{3}{*}{4} & Sep-2005 & Mala & Mala & Mala & Mala & Mala & Mala \\
\hline & Mar-2006 & Mala & Mala & Mala & Mala & Mala & Mala \\
\hline & Jul-2006 & Mala & Mala & Mala & Mala & Mala & Mala \\
\hline \multirow{3}{*}{5} & Sep-2005 & Mala & Regular & Mala & Mala & Mala & Mala \\
\hline & Mar-2006 & Mala & Mala & Mala & Mala & Mala & Mala \\
\hline & Jul-2006 & Mala & Mala & Mala & Mala & Mala & Mala \\
\hline
\end{tabular}


$\mathrm{Al}$ hacer uso de las dos fórmulas de agregación para el cálculo del ICA arrojaron resultados similares, siendo esto bueno debido a que no hay un criterio fuerte en la teoría que mencione la mejor forma de agregación para la elaboración de estos índices, por su frecuente uso en la elaboración de los ICAs y por la practicidad se decidió trabajar con la fórmula de agregación del promedio aritmético ponderado además de lo mencionado anteriormente.

Según estos resultados se presentó una similitud en todas las estaciones donde el ICA-General propuesto comparado con el ICANSF y el ICA-Orgánico comparado con el ICOMO presentaron las mismas conclusiones del estado de la calidad del agua. Además se observa que para todas las temporadas, la calidad es calificada en un rango de mala calidad, caso que no ocurre en la evaluación a partir del indicador ICOMO; esto indica que la propuesta ICANSF es la más estricta en la evaluación de la calidad el recurso hídrico.

Los resultados indican que las aguas residuales industriales y domésticas que llegan a la corriente son las causantes del deterioro de la calidad del recurso hídrico, el cual no es apto para consumo, recreación y contacto secundario de acuerdo al Decreto 3930/2010.

\section{CONCLUSIONES}

Se encontró que los niveles de $\mathrm{pH}$ son muy homogéneos en las estaciones, mientras que el Oxígeno Disuelto y la Turbiedad registraron la mayor variabilidad entre las estaciones, mostrando el impacto de las aguas residuales que recibe de los centros poblados, los cuales han impactado fuertemente en la calidad del agua. El índice de calidad del agua obtenido mediante la metodología propuesta en este artículo, mostró como resultado una gran similitud en comparación con los ICAs tradicionalmente conocidos.

Se encontraron formas alternativas y novedosas para evaluar la calidad del agua, utilizando una forma práctica y de sencillo análisis, obteniendo resultados muy similares, comparados con los demás indicadores existentes que llevan años de aplicación por parte de investigadores. 
En términos generales, este trabajo resalta la importancia y el uso de la estadística aplicada en la solución de un problema no trivial y de importancia ambiental, sin el uso de una teoría estadística sofisticada, teniendo en cuenta la experiencia y el recorrido de los grupos de investigación que cooperaron a dar su opinión. El índice de calidad del agua propuesto se deberá de ajustar a las condiciones ambientales del recurso hídrico estudiado, para que pueda ser usado de una forma adecuada.

En este trabajo se empleó una metodología diferente a las planteadas en trabajos e investigaciones anteriores para la construcción de los ICAs, donde hacen uso del análisis de componentes principales, tomando como punto de partida las encuestas realizadas a cuatro grupos de investigación en calidad del agua. Se encontraron diferencias de opinión, por tanto se seleccionó la curva que mejor representara el criterio global de los expertos, que es representada por la mediana; esto permitió tener una medida de tendencia central de estas curvas, obviando opiniones extremas de los expertos.

El indicador aquí plateado puede ser reformulado y debe ser validado mediante un monitoreo de calidad del agua en el que se incluyan las variables seleccionadas; es importante resaltar que los pesos asignados pueden ser modificados a medida que se conozca y comprenda la variación de cada parámetro a través de las estaciones y el tiempo. La gran carga contaminante proveniente de la industria azucarera y municipal, con valores superiores en 345mg/L de DQO, 135g/L de $\mathrm{DBO}_{5}$ y 2000 NMP/100mL de Coliformes Fecales, son la principal fuente de contaminación del cauce principal de la Subcuenca de Zanjón Oscuro que restringen el uso para consumo, contacto primario y secundario.

\section{AGRADECIMIENTOS}

Al grupo IREHISA de la Universidad del Valle, por el apoyo para realizar este trabajo. A la Corporación Autónoma Regional del Cauca, a la Universidad de Antioquia y a la Universidad del Valle por responder las encuestas realizadas. 


\section{REFERENCIAS}

Abbasi, S.A., (2002); Water quality indices, state of the art, Centre for Pollution Control \& Energy Technology, Pondicherry University, R. Venkataraman Nagar, Kalapet, Pondicherry.

Ball, R., Church, R., (1980); Water quality indexing and scoring, Journal of the Environmental Engineering Division, American Society of Civil Engineers, 106, EE4, 757-771.

CRC-FONAN., (2000); Monitoreo ambiental con énfasis en el recurso hídrico - Cuenca Cauca, Departamento del Cauca. Fase I. Calidad del Agua, Informe Final, Oficina de Investigaciones Ambientales, Convenio CRC-FONAN Nº 1287 de 1996.

Hair, J., Anderson, R., Tatham, R., Black, W., (1999); Análisis multivariante, $5^{\mathrm{a}}$ Edición, Prentice Hall.

Hernández, J., Ramírez, M.J., Ferri, C., (2004); Introducción a la minería de datos, Pearson-Prentice Hall.

Horton, R.K., (1965); An index number system for rating water quality, Journal of WPCF, 37, 1965.

IDEAM, (2004); Guía para el monitoreo de vertimientos, aguas superficiales y subterráneas, Instituto de Hidrología, Meteorología y Estudios Ambientales, Ministerio de Ambiente, Vivienda y Desarrollo Territorial - República de Colombia.

Liebman, H., (1969); Atlas of water quality: methods and practical conditions, R. Oldenbourgh, Munich.

Martínez, C., (2006); Estadística básica aplicada, 3 ed., Ecoe Ediciones, $406 \mathrm{p}$.

MAVDT, (2010); Decreto 3930 del 25 de Octubre de 2010, Por el cual se reglamenta parcialmente el Título I de la Ley 9 de 1979, así como el Capítulo II del Título VI - Parte III - Libro II del Decreto -Ley 2811 de 1974 en cuanto a usos del agua y residuos líquidos y se dictan otras disposiciones. Ministerio de Ambiente, Vivienda y Desarrollo Territorio.

NSF, (2006); National Sanitation Foundation, Consumer Information: Water Quality Index (WQI). En:

www.nsf.org/consumer/just_for_kids/wqi.asp Visitada: Marzo de 2008. 
Peña, D., (2002); Análisis de datos multivariantes, McGraw-Hill.

Ramírez, A., Viña, G., (1998); Cuatro índices de contaminación para caracterización de aguas continentales, formulación y aplicación, Ciencia Tecnología y Futuro, 1(3), 135-153.

Romero, R., Zúnica, R., (2008); Métodos estadísticos en ingeniería, Ed LIMUSA, México.

Samboni, N., Carvajal-Escobar, Y., (2007); A review of physical-chemical parameters as water quality and contamination indicators, Ing. Investig., Sep./Dec., 27(3), 172-181.

Samboní, N., (2009); Estimación del índice de escasez empleando los indicadores de calidad y contaminación del agua en la cuenca alta del Río Cauca, Tesis de Maestría, Programa Maestría en Ingeniería Sanitaria y Ambiental, Facultad de Ingeniería, Universidad del Valle.

Van Helmond, C., Breukel, R., (1997); Physico-Chemical water quality indices. Procedings of an International Works on Information Strategies in Water Management, Nunspeet, The Netherlands, 475-479. 